\title{
Images Directes de Fibrés en Droites Adjoints
}

\author{
par \\ Christophe MOUROUGAnE*
}

\begin{abstract}
Our main purpose is to study ampleness and positivity properties of the direct image $\varphi_{\star L} L$ of a holomorphic line bundle $L$ under a smooth morphism $\varphi: X \rightarrow Y$ between compact complex analytic manifolds. We show that in general the ampleness of $L$ does not imply that of the direct image $\varphi_{\star} L$ but only that of the direct image of the adjoint line bundle $\varphi_{\star}\left(K_{X / Y} \otimes L\right)$.
\end{abstract}

\section{\$1. Introduction}

L'objet de ce travail est l'étude des propriétés d'amplitude et de positivité sur $Y$ de l'image directe $\varphi_{\star} L$ d'un fibré en droites holomorphe $L$ par un morphisme lisse $\varphi: X \rightarrow Y$ entre variétés analytiques complexes compactes. Nous montrerons qu'en général l'amplitude de $L$ n'implique pas celle de l'image directe $\varphi_{\star} L$, mais seulement celle de l'image directe du fibré adjoint par le fibré canonique relatif $\varphi_{\star}\left(K_{X / Y} \otimes L\right)$.

Des résultats dans cette direction ont été démontrés par Fujita [Fuj] dans le cas où $Y$ est une courbe, par Kawamata [Kaw1] et Kollár [Kol] pour l'image directe du fibré canonique relatif sur les variétés projectives. Citons le résultat principal de Kawamata, dont la démonstration a été simplifiée par Kollár:

Théorème. Soit $\varphi: \quad X \rightarrow Y$ un morphisme surjectif entre variétés projectives lisses. Supposons que le diviseur de ramification soit à croisements normaux et qu'en dehors d'un ensemble de codimension 2 , les fibres de $\varphi$ soient réduites.

Alors, $\varphi \star K_{X / Y}$ est localement libre et numériquement effectif.

Les méthodes utilisées dans le cadre analytique conduisent à des propriétés de positivité en termes de tenseurs de courbure, a priori plus fortes que les

Communiqué par S. Mori, le 5, avril, 1996.

1991 Mathematics Subject Classification (s): 14F05, 32C17, 32J25.

* Institut Fourier, Université Grenoble I, B. P 74, 38402 Saint-Martin d'Hères, France.

Université Paris VI. Institut de Mathématiques, B. P. 247, 4, place Jussieu, 75252 Paris Cedex 05 , France.

e-mail: mourouga@math.jussieu. fr 
propriétés de positivité algébriques.

Nous obtenons des applications en direction de la conjecture de Griffiths reliant amplitude et positivité des fibrés vectoriels ( $[G$ riff $]$, problème 0.9 ). et pour la classification des variétés kählériennes compactes dont le fibré tangent est numériquement effectif [DPS].

Je tiens à remercier Michel Brion, Jean-Pierre Demailly et Laurent Manivel pour quelques discussions utiles dans la perspective de ce travail.

\subsection{Déffinitions}

Pour tout morphisme $f: X \rightarrow Y$ entre variétés algébriques ou analytiques complexes lisses, nous noterons $K_{X / Y}^{f}$ ou $K_{X / Y}$ le fibré en droites canonique relatif $K_{X} \otimes f^{2} K_{Y}^{-1}$. Si $f$ est un morphisme fini $K_{X / Y}$ est égal en tant que diviseur au lieu de ramification du morphisme $f$. Si $f$ est une submersion, $K_{X / Y}$ est égal au déterminant du faisceau des différentielles relatives $\Omega_{X / Y}$ défini par $\Delta^{\boldsymbol{L}}\left(\mathscr{I} / \mathscr{I}^{2}\right)$ où $\Delta: X \rightarrow X_{\times Y} X$ est le morphisme diagonal relatif à $f$ et $\mathscr{I}$ le faisceau d'idéaux de $\Delta(X)$ dans $X_{\times Y} X$.

Un fibré en droites holomorphe sur une variété analytique complexe compacte $X$ munie d'une métrique hermitienne $\omega$ de classe $C^{\infty}$ est dit numériquement effectif (nef) si pour tout $\varepsilon>0$, il peut être muni d'une métrique hermitienne de classe $C^{\infty}$ à courbure minorée par $-\varepsilon \omega$. Si $X$ est projective, cela revient à supposer que son degré sur toute courbe irréductible est positif ou nul.

Un fibré en droites holomorphe sur une variété $X$ est dit gros si sa dimension de Kodaira-litaka est égale à la dimension de $X$.

Un fibré vectoriel holomorphe $E$ est dit ample (resp. nef, resp. gros) si le fibré en droites canoniquement associé $\mathscr{O}_{E}(1)$ est ample (resp. nef, resp. gros) sur $\mathbb{P}(E)$ la variété des hyperplans de $E$. Nous renvoyons à [DPS] pour l'étude des propriétés des fibrés vectoriels nef. Précisons simplement.

Lemme. Un fibré vectoriel holomorphe $E$ sur une variété projective $X$ est nef si et seulement si pour toute application $g$ d'une courbe $C$ dans $X$, le degré sur $C$ de tout fibré en droites quotient de $g^{\star} E$ est positif ou nul.

Soit $f$ un morphisme lisse entre variétés analytiques complexes compactes. Un fibré vectoriel holomorphe est dit f-ample (resp. f-nef, resp. f-gros) si ses restrictions à toutes les fibres du morphisme $f$ sont des fibrés amples (resp. nef, resp. gros).

Un fibré vectoriel holomorphe $E$ sur une variété analytique complexe lisse $X$, est dit positif au sens de Griffiths s'il peut être muni d'une métrique hermitienne $h$ de classe $C^{\infty}$ dont la courbure $i c_{h}(E),(1,1)$-forme à valeurs dans le fibré des endomorphismes hermitiens de $E$, vérifie: pour tout $x \in X$ et pour 
tout $\xi \otimes v \in T_{x} X \otimes E_{x}$,

$$
\left.\left\langle\left(\xi, J_{x} \xi\right)\right\rfloor i c_{h}(E)_{x} v, v\right\rangle_{h} \geq 0
$$

où $J$ est la structure complexe sur $T X$.

\subsection{Sistude d'un exemple}

L'image réciproque d'un fibré vectoriel nef (par un morphisme quelconque) est un fibré vectoriel nef. La situation est différente pour les images directes.

Soit $\pi: X \rightarrow Y$ le revêtement cyclique ramifié le long d'un diviseur lisse et réduit $B$ donné par une section d'un fibré en droites globalement engendré $L^{k}$. Alors $\pi_{i \pi} \mathscr{O}_{x}=\bigoplus_{j=0}^{k-1} L^{-j}$ n'est pas nef; par contre puisque le fibré canonique relatif donné par la ramification est $K_{X / Y}=\pi^{\natural} L^{k-1}$, le faisceau localement libre $\pi_{\sharp} K_{X / Y}$ $=\pi_{\varkappa}\left(\mathscr{O}_{X}\right) \otimes L^{k-1}=\bigoplus_{j=0}^{k-1} L_{j}$ est nef.

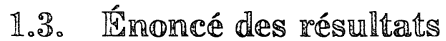

Au paragraphe 3 nous exposons une démonstration algébrique des résultats de positivité pour l'image directe par un morphisme lisse d'un fibré en droites nef et relativement gros adjoint par le fibré canonique relatif.

Théẹrème 1 (Version algébrique)。Soient $\varphi: X \rightarrow Y$ un morphisme surjectif lisse entre deux variétés projectives lisses, et $L \rightarrow X$ un fibré en droites ample (resp. numériquement effectif et $\varphi$-gros).

Alors $\varphi_{\text {↔ }}\left(K_{X / Y} \otimes L\right)$ est localement libre et ample (resp. localement libre et numériquement effectif).

Dans le cas d'un fibré semi-ample, ce résultat est une conséquence, par utilisation de revêtements cycliques, de théorèmes sur le caractère numériquement effectif de l'image directe du fibré canonique relatif ([Vie3] proposition 2.43).

En suivant un schéma de démonstration analogue, nous obtenons au paragraphe 4 une version kählérienne de ce résultat.

Théorème 2 (Version analytique). Soient $\varphi: X \rightarrow Y$ une submersion entre deux variétés kählériennes compactes lisses et $L \rightarrow X$ un fibré en droites numériquement effectif et $\varphi$-ample.

Alors $\varphi_{\star}\left(K_{X / Y} \otimes L\right)$ est localement libre et numériquement effectif.

Il suffit ici aussi de supposer que $L$ est seulement $\varphi$-gros. Mais la technique est alors plus difficile. La démonstration repose sur la construction de métriques hermitiennes à l'aide d'estimations $L^{2}$. Le théorème 2 montre en particulier le résultat suivant: 
Corollaire $\mathbb{1}[D P S]$. Soit $X$ une variété kählérienne compacte lisse dont le fibré tangent est numériquemment effectif. Alors, il existe un revêtement étale fini $\widetilde{X} \rightarrow X$ tel que, en notant $\alpha_{\tilde{X}}$ le morphisme d'Albanese de $\tilde{X}$, pour tout entier naturel $k$, $\alpha_{\tilde{X}_{\star}}\left(K_{\bar{X}_{\tilde{X}}}^{k}\right)$ est un fibré vectoriel numériquement effectif.

En ajoutant une hypothèse $(\mathrm{H})$ sur la variété de base, nous obtenons au paragraphe 5 une conclusion plus forte que celle du théorème 1 :

Hypothèse $(\mathrm{H})$. Il existe sur $Y$ une auto-application $\theta: Y \rightarrow Y$ et une métrique kählérienne $\omega$ telles que, pour un $\alpha>1,\left\{\theta^{\star} \omega\right\} \geq \alpha\{\omega\}$.

Ici $\{a\}$ désigne la classe de $i \partial \bar{\partial}$-cohomologie de la $(1,1)$-forme différentielle $a$. La notation $\{a\} \geq\{b\}$ signifie que pour tout $\varepsilon>0$, il existe une fonction $F$ de classe $C^{\infty}$ sur $Y$ telle que

$$
a \geq b+i \partial \bar{\partial} F-\varepsilon \omega .
$$

Théorème 3. Soient $X$ et $Y$ deux variétés projecives complexes lisses, $\varphi: X \rightarrow$ $Y$ une submersion, et $L \rightarrow X$ un fibré en droites ample. Supposons que l'hypothèse $(\mathrm{H})$ soit satisfaite sur $Y$.

Alors le fibré $\varphi\left(K_{X / Y} \otimes L\right)$ est strictement positif au sens de Griffiths (donc ample).

Un outil important de la démonstration est un procédé de régularisation des métriques continues sur un fibré vectoriel.

Le théorème 3 a pour corollaire le résultat suivant qui serait une conséquence de la conjecture de Griffiths.

Corollaire 2. Soit $Y$ une variété projective lisse dont un revêtement ramifié est une variété projective lisse vérifiant l'hypothèse $(\mathrm{H})$. Soit $E \rightarrow Y$ un fibré vectoriel ample sur $Y$. Alors pour tout entier naturel $k, S^{k} E \otimes \operatorname{det} E$ est strictement positif au sens de Griffiths.

Ce corollaire s'applique en particulier sur les variétés projectives lisses à courbure sectionnelle holomorphe constante positive ou nulle (i.e. l'espace projectif $\mathbb{P}^{n}$ et les variétés revêtues sans ramification par des variétés abéliennes [Ig 54]).

\subsection{Compléments sur l'hypothèse $(\mathbb{H})$}

L'hypothèse $(\mathrm{H})$ est satisfaite par

- les tores compacts: par image réciproque par la multiplication par 2 une métrique kählérienne est multipliée par 4.

- l'espace projectif: pour l'élévation au carré des coordonnées homogènes, l'image réciproque de $\mathscr{O}(1)$ est $\mathscr{O}(2)$.

- plus généralement les variétés toriques [Dan]: l'élévation au carrè des 
composantẹs dans les cartes affines $\mathbf{C}^{n}$ se recolle car les changements de cartes sont des mônomes de Laurent. L'effet de cette auto-application $\theta$ sur les fibrés en droites est déterminé par l'effet sur leurs restrictions au tore dense et donc par l'effet sur les caractères de ce tore. Si $L$ est un fibré en droites associé au caractère $\lambda$, le fibré $\theta^{\star} L$ est isomorphe à $L^{2}$ puisque le caractère $\theta^{\star} \lambda$ est égal à $\lambda^{2}$.

Cette hypothèse a été initialement considérée pour retrouver sur $\mathbf{C}$ une application analogue aux morphismes de Frobenius. Elle fournit d'autre part, dans certains contextes, un substitut au produit tensoriel de fibrés en droites.

\section{§2. Préliminaires}

Les lemmes suivants seront utiles dans la manipulation des faisceaux.

Lemme 1 ([Hart 2] exercice III. 8.3). Formule de projection:

Soit $f: X \rightarrow Y$ un morphisme de variétés, F un faisceau cohérent sur $X$, et $\mathscr{E}$ un faisceau localement libre de rang fini sur $Y$.

Alors pour tout $i \in \mathbb{N}$, les faisceaux images directes supérieures $\mathscr{R}^{i} f_{\star}\left(\mathscr{F} \otimes f^{\star} \mathscr{E}\right)$ et $\mathscr{R}^{i} f_{\star}(\mathscr{F}) \otimes \mathscr{E}$ sont naturellement isomorphes.

Lemme 2 ([Hart 2] proposition III. 9.3). Formule de changement de base:

Soit $f: X \rightarrow Y$ un morphisme de variétés et $\mathscr{F}$ un faisceau cohérent sur $X$. Soit $Y^{\prime}$ une variété et $\theta: Y^{\prime} \rightarrow Y$ un morphisme plat. Soit enfin $X^{\prime}:=X_{\times Y} Y^{\prime}$. On a donc le diagramme suivant:

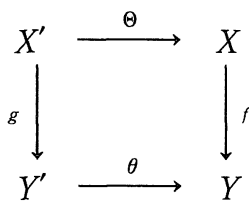

avec $\mathscr{F} \rightarrow X$.

Alors pour tout $i \in \mathbf{N}, \theta^{\star} \mathscr{R}^{i} f_{\star}(\mathscr{F})$ et $\mathscr{R}^{i} g_{\star}\left(\Theta^{\star} \mathscr{F}\right)$ sont naturellement isomorphes.

Avec les notations précédentes, les faisceaux de différentielles relatives $\Omega_{X^{\prime} / Y^{\prime}}$ et $\Theta^{\star}\left(\Omega_{X / Y}\right)$ sont isomorphes ([Hart2] proposition II. 8.10). Si de plus $f$, et par suite $g$ sont des submersions, $K_{X^{\prime} / Y^{\prime}}^{g}=\operatorname{det}\left(\Omega_{X^{\prime} / Y^{\prime}}\right)$ et $\Theta^{\star} K_{X / Y}^{f}$ sont isomorphes. On obtient ainsi une formule de changement de base pour les fibrés adjoints: pour tout $i \in \mathbf{N}$,

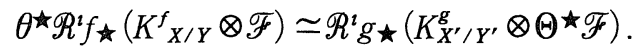

Cette formule peut aussi être obtenue à partir de la première formule de changement de base par application de la dualité de Serre sur les fibres de $f$ : puisque $f$ est supposée submersive, ses fibres sont lisses et leur faisceau dualisant coïncide avec la restriction du faisceau canonique relatif. 
Dans le cadre analytique, nous utiliserons les lemmes suivants:

Lemm 3. Soient $X$ une variété analytique complexe compacte et $p:(E, h) \rightarrow X$ un fibré vectoriel holomorphe hermitien. Soit $D=D^{\prime}+D^{\prime \prime}$ sa connexion de Chern. Soit $s$ une section holomorphe locale de $E$ au voisinage d'un point $x_{0}$ de $X$. Alors pour tout $x$ au voisinage de $x_{0}$ et $\xi \in T_{x} X$,

$$
\left.\left(\xi, J_{x} \xi\right)\right\rfloor i \partial \bar{\partial} \log \|s\|^{2} \geq \frac{\left.\left\langle\left(\xi, J_{x} \xi\right)\right\rfloor i c_{h}(E)_{x} s_{x}, s_{x}\right\rangle_{h}}{\left\|s_{x}\right\|^{2}}
$$

avec égalité si $D^{\prime} s=0$ au point $x$.

Lemme 4 ([Dem3], Lemme 4.4). Soient $X$ une variété analytique complexe compacte, $\gamma$ une $(1,1)$-forme réelle sur $X$, et $p:(E \dot{w}, h) \rightarrow X$ un fibré vecioriel holomorphe hermitien.

Alors



où $\log h$ est considérée comme fonction sur l'espace total $E^{\vec{\mu}}$. Ici $\geq_{\text {Griff désigne la }}$ positivité au sens de Griffiths.

Lemane 5. Soient $u_{1}, \ldots, u_{p}$ des fonctions plurisousharmoniques sur une variété analytique complexe $X$. Alors, $\log \left(e^{u_{1}}+\cdots+e^{u_{p}}\right)$ est plurisonsharmonique sur $X$.

Ces deux derniers lemmes montrent en particulier que la "somme" de métriques à courbure négative est encore à courbure négative.

\section{§3. Démonstration du Théorème Algébrịune}

Ici, $X$ et $Y$ sont deux variétés projectives lisses connexes de dimension respective $n$ et $m$. Le morphisme $\varphi: X \rightarrow Y$ est supposé lisse. Le cas d'un fibré en droites ample se réduit au cas d'un fibré en droites nef et relativement gros par soustraction de l'image réciproque sur $X$ d'un $\mathbb{Q}$-diviseur ample assez petit sur $Y$ et par un revêtement ramifié fini de $Y$. On suppose donc que $L \rightarrow X$ est un fibré en droites nef et $\varphi$-gros.

\subsection{Première étape: $\varphi_{\text {t }}\left(K_{X / Y} \otimes L\right)$ est localement libre}

Puisque $\varphi$ est un morphisme lisse, le théorème d'annulation de KawamataViehweg [Kaw2] [Viel], s'applique sur les fibres de $\varphi$ (qui sont toutes réduites lisses et projectives) et donne

$$
\forall j>0, \quad \mathscr{R}^{j} \varphi_{\text {々 }}\left(K_{X / Y} \otimes L\right)=0 .
$$

Par platitude de $\varphi$, en désignant par $F_{y}$ la fibre de $\varphi$ en $y$, 


$$
y \mapsto \operatorname{dim} H^{0}\left(F_{y}, K_{F y} \otimes L\right)
$$

est localement constante sur $Y$. Par un théorème de Grauert ([Hart2] corollaire III. 12.9) le faisceau $\varphi_{\text {出 }}\left(K_{X / Y} \otimes L\right)$ est localement libre. La même démonstration s'applique au cas d'une submersion entre variétés kählériennes compactes et d'un fibré relativement gros.

\subsection{Denxième étape:}

Soit $G \rightarrow Y$ un fibré en droites très ample sur $Y$. On montre que pour toute donnée $(X, \varphi, L)$ vérifiant les hypothèses du théorème 1 , si $E$ désigne le fibré vectoriel associé au faisceau localement libre $\varphi_{\hookleftarrow}\left(K_{X / Y} \otimes L\right)$, alors $E \otimes K_{Y} \otimes G^{m+1}$ est globálement engendré.

A cette fin, on dispose du critère de Castelnuovo-Mumford [Mumf].

Lemme 6. Soit $Y$ une variété projective lisse munie d'un fibré en droites très ample $G$. Soit $E \rightarrow Y$ un fibré vectoriel. Si les groupes de cohomologie $H^{\imath}\left(Y, E \otimes G^{-\imath}\right)$ sont nuls pour tout $i>0$, alors $E$ est globalement engendré.

Ce lemme se démontre en vérifiant que les morphismes de restriction aux intersections transverses décroissantes de diviseurs de $|G|$ sont surjectifs.

Il suffit donc de vérifier l'annulation, pour $0<i \leq m$, de

$$
\begin{aligned}
H^{\imath}\left(Y, E \otimes K_{Y} \otimes G^{m+1-\imath}\right) & =H^{\imath}\left(Y, \varphi_{\text {出 }}\left(K_{X} \otimes L\right) \otimes G^{m+1-\imath}\right) \\
& =H^{\imath}\left(X, K_{X} \otimes L \otimes \varphi^{\downarrow} G^{m+1-\imath}\right) .
\end{aligned}
$$

La deuxième égalité a lieu car les images directes supérieures $\mathscr{R}^{j} \varphi_{\star}\left(K_{X} \otimes L\right)=$ $K_{Y} \otimes \mathscr{R}^{\jmath} \varphi_{\text {々 }}\left(K_{X / Y} \otimes L\right)$ sont nulles.

Maintenant, puisque $L$ et $G$ sont nef, $L \otimes \varphi^{\star} G^{m+1-\imath}$ l'est aussi. Puisque $L$ est

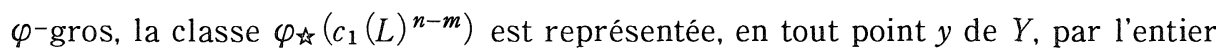
strictement positif $\int_{F_{y}} c_{1}\left(L_{\mid F_{y}}\right)^{n-m}$ où $F_{y}$ est la fibre de $\varphi$ en $y$. Puisque de plus $G$ est très ample,

$$
\int_{X}\left(c_{1}\left(L \otimes \varphi^{\star} G^{m+1-\imath}\right)\right)^{n}=\sum_{k=0}^{m} C_{n}^{k}(m+1-i)^{k} \int_{Y} \varphi_{\star}\left(c_{1}(L)^{n-k}\right) \wedge c_{1}(G)^{k}
$$

est une somme de termes positifs dont le dernier est strictement positif. La dimension numérique et par suite la dimension de Kodaira-Iitaka de $L \otimes \varphi^{\star} G^{m+1-1}$ sont donc maximales: $L \otimes \varphi^{\star} G^{m+1-\imath}$ est gros. On applique alors le théorème de Kawamata-Viehweg pour conclure.

\subsection{Troisième étape:}

On déduit de l'étape précédente que pour tout $s \in \mathbf{N}, E^{\otimes_{s}} \otimes K_{Y} \otimes G^{m+1}$ est globalement engendré. 
Pour cela, on considère une construction inspirée de E. Viehweg ([Vie2] Lemma 3.5). Soit $X^{(s)}:=X \times{ }_{\varphi} X \times{ }_{\varphi} \cdots \times{ }_{\varphi} X$ le produit fibré de $s$ copies de $X$. On note $\varphi_{i}^{(s)}: X^{(s)} \rightarrow X_{i}$ la projection sur le $i^{\text {ième }}$ facteur, $\varphi_{i}: X_{i}=X \rightarrow Y$ I'application $\varphi$ et $L_{i} \rightarrow X_{i}$ le fibré en droites $L$. Puisque $X, Y$ et $\varphi$ sont lisses le produit fibré $X^{(s)}$ est une variété lisse. L'application naturelle $\varphi^{(s)}: X^{(s)} \rightarrow Y$ est lisse.

$L^{(s)}:=\bigotimes_{i=1}^{s} \varphi_{i}^{(s)} L_{i}$ est nef et $\varphi^{(s)}$-gros.

Afin d'exploiter le résultat de l'étape précédente, il suffit de remarquer que, par application des formules de projection et de changement de base pour les fibrés adjoints

$$
\varphi_{\downarrow}^{(s)}\left(K_{X^{(s)} / Y} \otimes L^{(s)}\right)=\left(\varphi_{\star}\left(K_{X / Y} \otimes L\right)\right){ }^{\otimes_{s}} .
$$

\subsection{Quatrième étape: $\mathbb{E}$ est nef}

Soit $\pi: \mathbb{P}(E) \rightarrow Y$ la variété des hyperplans de $E$. Sur $\mathbb{P}(E)$, on dispose d'une application surjective entre fibrés

$$
\pi^{\star} E^{\otimes s} \rightarrow \pi^{\star} S^{s} E \rightarrow \mathscr{O}_{E}(s)
$$

obtenue par la symétrisation suivie de l'application d'évaluation fibre à fibre $\operatorname{sur} \mathfrak{O}_{E}(s)$. On en déduit une application surjective

$$
\pi^{\star}\left(E^{\otimes_{s}} \otimes K_{Y} \otimes G^{m+1}\right) \rightarrow \mathscr{O}_{E}(s) \otimes \pi^{\star}\left(K_{Y} \otimes G^{m+1}\right)
$$

qui permet de munir $\mathscr{O}_{E}(s) \otimes \pi^{\star}\left(K_{Y} \otimes G^{m+1}\right)$ d'une métrique à courbure positive et donc $\mathscr{O}_{E}(1)$ d'une métrique $h^{(s)}$ dont la courbure vérifie

$$
i c_{h^{(s)}}\left(\mathscr{O}_{E}(1)\right) \geq-\frac{1}{s} \pi^{\star} i c_{H}\left(K_{Y} \otimes G^{m+1}\right)
$$

où $H$ est une métrique hermitienne de classe $\mathscr{C}^{\infty}$ fixée sur $K_{Y} \otimes G^{m+1}$. Les fibrés $\mathfrak{O}_{E}(1)$ et $E$ sont donc nef.

\section{§4. Démonstration du Théorème Analytique}

Le morphisme $\varphi: X \rightarrow Y$ est une submersion entre variétés kählériennes compactes lisses et $L \rightarrow X$ est un fibré en droites nef et $\varphi$-ample.

\subsection{Métriques sur les fibrés en droites nef et relativement amples}

On cherche à traduire en termes de métriques l'hypothèse sur le fibré en droites.

Lemme 7. Soient $\varphi: X \rightarrow Y$ une application surjective entre variétés kählériennes compactes lisses et $L \rightarrow Y$ un fibré en droites sur $X$ nef et $\varphi$-ample. Soit 
$\omega$ une métrique kählérienne sur $Y$.

Alors, pour tout $\varepsilon>0$, il existe une métrique hermitienne $H$ de classe $\mathscr{C}^{\infty}$ sur $L$ telle que $i c_{H}(L)+\varepsilon \varphi^{\star} \omega$ soit une métrique kählérienne sur $X$.

Démonstration. Puisque $L$ est $\varphi$-ample et que $Y$ est compacte, les estimations $L^{2}$ pour l'opérateur $\bar{\partial}$ fournissent une puissance $p$-ième $L^{p}$ de $L(p \gg 0)$ telle que les fibres de $\varphi$ se plongent dans des espaces projectifs grâce à $L^{p}$ : en chaque point $y \in Y$, il faut que la courbure de $L_{\mid \varphi^{-1}(y)}^{p}$ compense certains poids logarithmiques, que l'on peut choisir dépendant continument de $y$. On obtient alors le diagramme suivant:

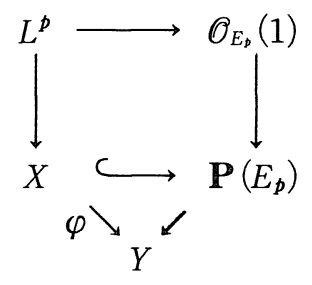

avec $E_{p}:=\varphi_{\star}\left(L^{p}\right)$ localement libre.

On fixe une métrique sur $E_{p}$. Pour la métrique $h$ induite sur $L$, il existe un réel $A>0$ tel que $i c\left(E_{p}\right) \geq-p A \omega \otimes \operatorname{Id}_{E_{p}}$. Par suite, $i c_{h}(L)+(A+1) \varphi^{\star} \omega$ est une métrique kählérienne sur $X$. Comme $L$ est nef, pour tout $\varepsilon>0$, il existe une métrique $h_{\varepsilon}$ sur $L$ telle que

$$
i c h_{\varepsilon}(L) \geq-\varepsilon\left(i c_{h}(L)+(A+1) \varphi^{\star} \omega\right) .
$$

La métrique $\tilde{h}:=\left(h_{\varepsilon} h^{\varepsilon}\right)^{1 / 1+\varepsilon}$ est alors telle que $i c_{\tilde{h}}(L)+\varepsilon(A+1) \varphi^{\star} \omega$ soit une métrique kählérienne sur $X$.

\subsection{Démonstration du théorème analytique}

\subsubsection{Première étape: Construction de métriques sur les images directes}

La proposition suivante fournit dans le cadre kählérien, l'argument analogue au fait que, dans un cadre projectif, pour toute donnée $(X, \varphi, L)$ satisfaisant les hypothèses du théorème 1 , le fibré $K_{Y} \otimes G^{m+1}$ ne dépendant que de $Y$ suffit à rendre $\varphi_{\star}\left(K_{X / Y} \otimes L\right) \otimes K_{Y} \otimes G^{m+1}$ globalement engendré (Deuxième étape).

Proposition 1. Soit $\varphi: X \rightarrow Y$ une submersion entre variétés kählériennes compactes et $L \rightarrow X$ un fibré en droites sur $X$ nef et $\varphi$-ample. Soit $\omega$ une métrique kählérienne sur $Y$. Alors il existe une constante $A$ ne dépendant que de $\omega$ et de $Y$, telle que pour toute donnée $(X, \varphi, L)$ vérifiant les hypothèses du théorème 2 , on peut munir $\varphi_{\star}\left(K_{X / Y} \otimes L\right)$ d'une métrique hermitienne $H$ de classe $\mathscr{C}^{\infty}$ vérifiant 


$$
i c_{H}\left(\varphi_{\text {㟧 }}\left(K_{X / Y} \otimes L\right)\right) \geq_{\text {Griff }}-A \omega \otimes \operatorname{Id}_{\varphi_{\varphi^{\prime}}\left(K_{X / Y} \otimes L\right)} .
$$

Démonstration. On utilise les méthodes de construction de métriques par estimations $L^{2}$ développées dans [Dem3].

Puisque $L$ est nef et $\varphi$-ample, le lemme 7 fournit une métrique sur $L$ telle que $w:=i c(L)+\varphi^{\vec{r}} \omega$ soit une métrique kählérienne sur $X$. Soit $\left(U_{j}\right)$ un recouvrement de $Y$ par des boules relativement compactes dans des cartes. Soit $v$, une fonction sur $U_{j}$ quadratique en les coordonnées, bornée, et vérifiant

$$
i \partial \bar{\partial} v_{j}-i c_{\omega}\left(K_{Y}^{-1}\right) \leq-\omega .
$$

On définit

$$
\mathscr{H}_{j}:=\left\{s \in H^{0}\left(\varphi^{-1}\left(U_{J}\right), K_{X / Y} \otimes L\right) / \int_{\varphi^{-1}(L,)}|s|_{K_{X / Y} \otimes L}^{2} e^{v, \circ \varphi} d V_{w}<+\infty\right\} .
$$

où la métrique sur $K_{X / Y}=K_{X} \otimes \varphi^{\text {tr }} K_{Y}^{-1}$ est déduite des métriques $w$ et $\omega$. Les poids sont tels que

$$
i c\left(K_{X}^{-1}\right)+i c\left(K_{X / Y} \otimes L\right)-\varphi^{\natural} i \partial \bar{\partial} v_{j} \geq i c(L)+\varphi^{\natural} \omega=w .
$$

Ce calcul met en évidence la nécessité d'adjoindre $K_{X / Y}$ à $L$ pour obtenir des estimations qui ne dépendent que de la base $Y$.

Pour $y \in U_{j}$ et $\xi \in E_{y}^{\text {年 }}$, soit la forme linéaire

$$
\begin{aligned}
\Xi: \mathscr{H}_{j} & \rightarrow \mathbb{C} \\
s & \mapsto s(y) . \xi
\end{aligned}
$$

où on considère $s \in H^{0}\left(U_{j}, E\right)$ avec $E_{y}$ et $E_{y}^{\not z}$ en dualité naturelle. On note $\|\xi\|$, la norme de la forme linéaire $\Xi$. En choisissant une base hilbertienne de $\mathscr{H}$, on constate que $\log \|\|^{2}$ est une fonction plurisousharmonique sur l'espace total $E_{U_{U} \text {. }}$ Soit $\left(U_{j}^{\prime}\right)$ et $\left(U_{j}^{\prime \prime}\right)$ deux recouvrements de $Y$ par des boules telles que $U_{j}^{\prime} \subset \subset U_{j}^{\prime \prime} \subset \subset U_{j}$ et $\theta_{j}$ une fonction positive de classe $\mathscr{C}^{\infty}$ à support dans $U_{j}^{\prime \prime}$, partout inférieure à 1 et identiquement égale à 1 sur $U_{j}^{\prime}$. Pour $y \in U_{j}$ et $\xi \in E_{y}^{\text {rz }}$, on pose

$$
\|\xi\|^{2}:=\sum \theta_{j}^{2}(y)\|\xi\|_{j}^{2}
$$

La fin de la démonstration consiste à montrer que la métrique ainsi définie (sur $E^{\star}$ et par dualité sur $E$ ) satisfait les conditions requises dans la proposition 1. Pour ceci, il suffit d'appliquer le lemme suivant qui permet de contrôler la perte de positivité due aux recollements des métriques \|\|$_{j}$.

Lemme 8 ([Dem3] lemme 3.5). Soit $X$ une variété complexe munie d'une $(1,1)$-forme positive $\Omega$. Soit $V_{j}^{\prime} \subset V_{j}^{\prime \prime} \subset V_{j}$ des recouvrements localement finis de $X$ par des ouverts relativement compacts. Soit $\theta_{j}$ une fonction positive de classe $\mathscr{C}^{\infty} \grave{a}$ 
support dans $V_{j}^{\prime \prime}$, partout inférievire à 1 et identiquement égale à 1 sur $V_{j}^{\prime}$. Soit $B_{j}$ des constantes telles que

$$
i\left(\theta_{j} \partial \bar{\partial} \theta_{j}-\partial \theta_{j} \wedge \bar{\partial} \theta_{j}\right) \geq-B_{j} \Omega \text { sur } V_{j}^{\prime \prime} \backslash V_{j}^{\prime}
$$

Soit $w_{j}$ une fonction presque pi,ursonsharmonique sur $V$, telle que, $i \partial \bar{\partial} w_{j} \geq \gamma$ pour une

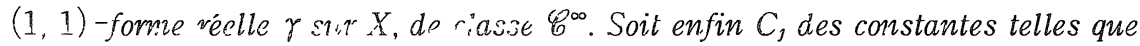

$$
\forall x \in V_{j}^{\prime \prime} \backslash V_{j}^{\prime}, w_{j}(x) \leq C_{j}+\sup _{k \neq j V_{k}^{\prime} \ni x} w_{k}(x) .
$$

Alors la fonction $w:=\log \left(\sum \theta_{j}^{2} e^{w_{j}}\right)$ est presque plurisousharmonique, et son hessien . Érifie

$$
i \partial \bar{\partial} w \geq \gamma-2\left(\sum \mathbb{1}_{V^{\prime}, \backslash V^{\prime}} B_{j} e^{C_{1}}\right) \Omega
$$

On montre d'abord l'existence d'une constante $C$ ne dépendant que de $Y$ telle gue

$$
\log \|\xi\|_{j}^{2} \leq C+\log \|\xi\|_{k}^{2} \operatorname{sur} p^{-1}\left(\left(U_{j}^{\prime \prime} \backslash U_{j}^{\prime}\right) \cap U_{k}^{\prime}\right) \subset E^{2},
$$

où $p: E^{r} \rightarrow Y$ est lá projection naturelle.

Soit donc $y \in\left(U_{j}^{\prime \prime} \backslash U_{j}^{\prime}\right) \cap U_{k}^{\prime}$ et $\xi \in E_{y}^{\varkappa}$. Soit $h_{j} \in \mathscr{H}_{j}$ telle que $\left\|h_{j}\right\|_{\mathscr{H}},=1$ et $|h,(y) . \xi|=\|\xi\|_{j}$. Il existe une constante $c_{0}$ ne dépendant que des recouvrements de $Y$, indépendante de $y \in Y$ et une fonction $\chi$ de classe $\mathscr{C}^{\infty}$ à support compact dans $U_{j} \cap U_{k}$, égale à 1 sur un voisinage $V_{y}$ de $y$ et telle que $|\bar{\partial} \chi| \leq c_{0}$.

La fonction $\phi:=(2 \operatorname{dim} Y) \log |\varphi-y| \operatorname{sur} \varphi^{-1}\left(U_{k}\right)$ est majorée sur $\varphi^{-1}\left(U_{k}\right)$ et minorée en dehors de $V_{y}$ par des constantes ne dépendant que des recouvrements de $Y$. Puisque $\varphi$ est une submersion, on a

$$
\int_{\varphi^{-1}\left(U_{k}\right)}|f|^{2} e^{-\phi} d V_{w}<+\infty \Rightarrow f \equiv 0 \operatorname{sur} \varphi^{-1}(y) .
$$

$\varphi^{-1}\left(U_{k}\right)$ est faiblement pseudo-convexe,

$\int_{\varphi^{-1}\left(U_{k}\right)}\left|\bar{\partial}\left(\chi h_{\jmath}\right)\right|^{2} e^{v_{k} \cdot \varphi-\psi} d V_{w}<+\infty$,

$i c\left(K_{X}^{-1}\right)+i c\left(K_{X / Y} \otimes L\right)-\varphi^{\star} i \partial \bar{\partial} u_{k}+i \partial \bar{\partial} \psi \geq w$.

L'application des estimations $L^{2}$ de Hörmander fournit donc une section $f_{k} \in \mathscr{C}^{\infty}\left(\varphi^{-1}\left(U_{k}\right), K_{X / Y} \otimes L\right)$ telle que

$$
\begin{aligned}
& \cdot \bar{\partial}\left(f_{k}-\chi h_{j}\right)=0 \\
& \cdot f_{k} \equiv 0 \operatorname{sur} \varphi^{-1}(y) \\
& \cdot \int_{\varphi^{-1}\left(U_{k}\right)}\left|f_{k}\right|^{2} e^{v_{k}^{\circ} \varphi-\psi} d V_{w} \leq c_{1} \int_{\varphi^{-1}\left(U_{k}\right)}\left|\bar{\partial}\left(\chi h_{j}\right)\right|^{2} e^{v_{\kappa^{\circ}} \varphi-\psi} d V_{w} .
\end{aligned}
$$

On obtient alors, en utilisant les bornes de $\phi$ et le fait que $\left|v_{j}-v_{k}\right|$ est majorée par une constante ne dépendant que de $Y$ 
Par suite

$$
\left\|f_{k}-\chi h_{j}\right\|_{\mathscr{H}_{k}} \leq c_{2}\left\|h_{j}\right\|_{\mathscr{H}}=c_{2}
$$

$$
\|\xi\|_{k} \geq \frac{\left|\left(f_{k}-\chi h_{j}\right)(y) \cdot \xi\right|}{\left\|f_{k}-\chi h_{j}\right\| \mathscr{H}_{k}} \geq \frac{\left|h_{j}(y) \cdot \xi\right|}{c_{2}} \geq \frac{\|\xi\|_{j}}{c_{2}} .
$$

D'où l'existence de la constante $C$.

En outre, il existe une constante $B$ ne dépendant que de $Y$ telle que

$$
i \theta_{j} \partial \bar{\partial} \theta_{j}-i \partial \theta_{j} \wedge \bar{\partial} \theta_{j} \geq-B \omega
$$

et donc

$$
i\left(\theta_{j}^{\circ} p\right) \partial \bar{\partial}\left(\theta_{j}^{\circ} p\right)-i \partial\left(\theta_{j}^{\circ} p\right) \wedge \bar{\partial}\left(\theta_{j} \circ p\right) \geq-B p^{\star} \omega .
$$

Le lemme de recollement 8 fournit donc

$$
i \partial \bar{\partial} \log \|\xi\|^{2} \geq p^{\star}\left(-2 B e^{C}\left(\sum_{j} 1 \mathrm{I}_{U^{\prime \prime} \backslash \bigcup^{\prime}}\right) \omega\right) \geq-A p^{\star} \omega
$$

pour une constante $A$ ne dépendant que de $Y$.

Le lemme 4 permet alors de conclure

$$
i c\left(\varphi_{\star}\left(K_{X / Y} \otimes L\right)\right) \geq_{\text {Griff }}-A \omega \otimes \operatorname{Id}_{\varphi_{\star}\left(K_{X / Y} \otimes L\right)} .
$$

\subsubsection{Deuxième étape:}

La construction de E. Viehweg des produits fibrés et la proposition 1 permettent de munir $E^{\otimes_{s}}$ d'une métrique hermitienne de classe $\mathscr{C}^{\infty}$ dont la courbure vérifie:

$$
i c\left(E^{\otimes s}\right) \geq_{\text {Griff }}-A \omega \otimes I d .
$$

\subsubsection{Troisième étape:}

On utilise l'application surjective $\pi^{\star} E^{\otimes_{s}} \rightarrow \mathscr{O}_{E}(s)$ pour munir $\mathscr{O}_{E}(1)$ d'une métrique hermitienne de classe $\mathscr{C}^{\infty}$ dont la courbure vérifie:

$$
i c\left(\mathscr{O}_{E}(1)\right) \geq-\frac{A}{s} \pi^{\star} \omega .
$$

Le fibré $E$ est donc nef.

\section{§5. Démonstration du Théorème de Positivité au Sens de Griffiths}

\subsection{Transport de la positivité par morphisme fini}

On montre d'abord que, comme l'amplitude ([Hart1] Proposition 4.3), la positivité au sens de Griffiths est conservée par morphisme fini. Comme le montrent les lemmes 4 et 5 , il est plus naturel d'étudier le transport de la négativité au sens de Griffiths.

Proposition 2. Soit $F: X^{\prime} \rightarrow X$ un morphisme fini surjectif entre variétés 
kählériennes compactes. Soient $\omega$ une métrique kählérienne sur $X$ et $p: E \rightarrow X$ un fibré vectoriel sur $X$. Soit $\nu$ un réel.

Alors

$$
i c\left(F^{\star} E\right)<_{\text {Griff }}-\nu F^{\star} \omega \otimes \mathrm{Id} \Longleftrightarrow i c(E)<_{\text {Griff }}-\nu \omega \otimes \text { Id }
$$

Démonstration. L'implication $\Longleftarrow$ est démontrée dans [Fran]: il suffit de corriger la métrique image réciproque aux points où la différentielle de $F$ s' annule.

Pour l'autre implication, on considère une métrique hermitienne $h$ de classe $C^{\infty}$ sur $F^{\star} E$ telle que pour $\alpha>0$ assez petit

$$
i c_{h}\left(F^{\star} E\right)<_{\text {Griff }}-(\nu+\alpha) F^{\star} \omega \otimes \operatorname{Id}_{F^{\star} E} .
$$

On munit $E$ de la métrique trace $h_{0}$ définie par

où $T$ est défini par

$$
\forall x \in X, \forall v \in E_{x}, h_{0}(x)(v)=\frac{1}{\operatorname{deg} F} \sum_{\substack{F\left(x^{\prime}\right)=x \\ T V=v}} h\left(x^{\prime}\right)(V)
$$

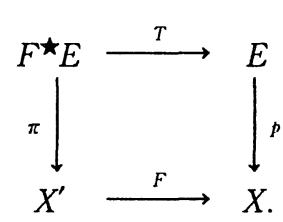

La métrique hermitienne $h_{0}$ sur $E$ est continue sur $X$, de classe $C^{\infty}$ en dehors du lieu de ramification de $F$.

On montre maintenant l'inégalité de courbure

$$
i \partial_{E} \bar{\partial}_{E} \log h_{0}>(\nu+\alpha) p^{\star} \omega \text { au sens des courants. }
$$

Puisque la métrique $h_{0}$ est continue, il suffit d'étudier sa courbure en dehors du lieu de ramification de $F$. Soit $x_{0} \in X$ en dehors du lieu de ramification de $F$. Soit $\left(F_{j}^{-1}\right)_{1 \leqq j \leqq \operatorname{deg} F}$ (resp. $\left(T_{j}^{-1}\right)_{1 \leqq j \leqq \operatorname{deg} F}$ ) des inverses de $F$ (resp. $T$ ) définis sur un voisinage ouvert de $x_{0}$. Alors au voisinage de $x_{0}$,

$$
\log h_{0}(x)(v)=\log \left(\frac{1}{\operatorname{deg} F} \sum_{j=1}^{\operatorname{deg} F} h\left(F_{j}^{-1}(x)\right)\left(T_{j}^{-1} v\right)\right) .
$$

On choisit des repères $h$-normaux aux voisinages des points $F_{j}^{-1}\left(x_{0}\right)$. Ainsi un calcul analogue à celui de la démonstration du lemme 5 permet d'obtenir l'inégalité de courbure souhaitée.

On remarque que cette inégalité permet ici de définir la connexion de Chern du fibré hermitien continu $\left(E, h_{0}\right)$.

On explicite maintenant le procédé de régularisation de la métrique 
hermitienne $h_{0}$. Puisqu'il s'agit de fonctions à valeurs matricielles, le procédé de Richberg de régularisation des fonctions plurisousharmoniques continues à l'aide de fonctions maximum ne s'applique pas. La méthode proposée ici résout la difficulté par utilisation du transport parallèle.

Lemme 9. Soient $(X, \omega)$ une variété hählérienne compacte et $p: E \rightarrow X$ un fibré vectoriel sur $X$. Soit $h_{0}$ une métrique hermitienne continue sur $E$ telle que, pour un réel $\nu$, et un réel $\alpha>0$

$$
i \partial_{E} \bar{\partial}_{E} h_{0}>(\nu+\alpha) h_{0} p^{\star} \omega \text { au sens des courants. }
$$

Alors il existe sur $E$ une métrique hermitienne $h$ de classe $C^{\infty}$ telle que

$$
i c_{h}(E)<_{\text {Griff }}-\nu \omega \otimes \operatorname{Id}_{E} .
$$

Remarque. Le procédé de régularisation et le résultat sont valables pour un majorant non nécessairement décomposable. Il faut alors modifier la démonstration en utilisant le lemme 3 au lieu du lemme 4.

Démonstration. On étend au cas de fonctions continues à valeurs matricielles la méthode de régularisation développée dans [Dem1].

Soit $H$ une métrique hermitienne de classe $C^{\infty}$ sur $E, D^{H}$ la connexion de Chern associée et $\tau:=\tau^{H}$ le transport parallèle relatif à $D^{H}$. Soit $\chi: \mathbb{R} \rightarrow \mathbb{R}$ l'application de classe $C^{\infty}$ définie par

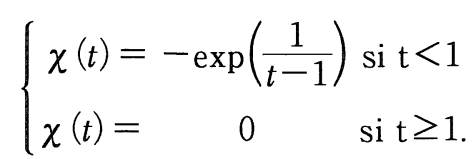

On désigne $\operatorname{par} C:=\int_{\xi \in \mathbb{C}^{n}} \chi^{\prime}\left(|\xi|^{2}\right) d \lambda(\xi)$ où $n$ est la dimension $\operatorname{de} X$.

Ces données permettent de définir par convolution une suite de métriques hermitiennes sur $E$ de classe $C^{\infty}$ : pour tout $\varepsilon>0, z \in X, V \in E_{z}$, on pose

$$
h_{\varepsilon}(z)(V):=\frac{1}{C \varepsilon^{2 n}} \int_{\xi \in T_{z} X} \chi^{\prime}\left(\frac{\|\xi\|_{\omega}^{2}}{\varepsilon^{2}}\right) h_{0}\left(\exp _{z} \xi\right)\left(\tau_{z, \xi}(V)\right) d \lambda_{\omega}(\xi),
$$

où $\tau_{z, \xi}$ désigne le transport parallèle relatif à la connexion $D^{H}$ le long de la géodésique $\gamma:[0,1] \rightarrow X, t \mapsto \exp _{z} t \xi$.

On commence par montrer que $h_{\varepsilon}$ est de classe $C^{\infty}$.

Soient $z_{0}$ un point de $X$ et $(u)=\left(u_{1}, \ldots, u_{n}\right)$ un système de coordonnées holomorphes sur $X$ au voisinage de $z_{0}$ tel que

$$
\omega_{j k}(u)=\delta_{j k}-\sum_{l_{p}} c_{j k l p} u \bar{u}_{p}+O\left(|u|^{3}\right),
$$

où $\left(c_{j k l p}\right)$ est le tenseur de courbure de $(T X, \omega)$ et $\delta_{j k}$ le symbole de Kronecker. 
Un tel système de coordonnées est dit $\omega$-géodésique en $z_{0}$. Son existence en tout point de la variété $X$ est équivalente au caractère kählérien de la forme $\omega$. On pose:

$$
\begin{array}{ll}
u_{k}:=u_{k}\left(\exp _{z} \xi\right), & z_{k}:=u_{k}(z), \\
v_{k}:=u_{k}-z_{k}, & \xi_{k}:=d_{z} u_{k} . \xi
\end{array}
$$

Dans tous les calculs qui suivront, $\left(u_{k}\right)$ et $\left(z_{k}\right)$ seront considérés comme deux $n$-uplets indépendants de variables indépendantes. L'espace total de $T X$ qui paramètre les petites géodésiques est de dimension $2 n$. La notation $v_{k}$ est une écriture condensée de $u_{k}-z_{k}$.

Puisque $(u)$ est un système de coordonnées $\omega$-géodésique, il existe un système $(\eta)=\left(\eta_{1}, \ldots, \eta_{n}\right)$ orthonormé $C^{\infty}$ de coordonnées sur les fibres de $T X$, au voisinage de $z_{0}$, tel que

$$
\|\xi\|_{\omega}^{2}=\sum\left|\eta_{\imath}\right|^{2}=|\eta|^{2}
$$

avec

$$
\eta_{k}=\xi_{k}-\frac{1}{2} \sum_{j l p} c_{j k l p} z_{l} \overline{z_{p}} \xi_{j}+O\left(|z|^{3}\right) \cdot \xi
$$

Pour transformer les variables $\eta$ en variables $z$ et $u$, on utilise le développement limité de l'exponentielle ([Dem1] lemme 8.2):

$$
u_{k}=z_{k}+\xi_{k}+\frac{1}{2} \sum_{j l p} c_{j k l p}\left(\bar{z}_{p}+\frac{\bar{\xi}_{p}}{3}\right) \xi_{j} \xi_{l}+O\left(|z|^{2}+|\xi|^{2}\right)|\xi|^{2}
$$

Ainsi

et

$$
\xi_{k}=v_{k}-\frac{1}{2} \sum_{j l p} c_{j k l p}\left(\overline{z_{p}}+\frac{\overline{v_{p}}}{3}\right) v_{j} v_{l}+O(|u|+|z|)^{4}
$$

$$
\eta_{k}=v_{k}-\frac{1}{2} \sum_{j l p} c_{j k l p}\left(\overline{z_{p}} v_{j} u_{l}+\frac{1}{3} \bar{v}_{p} v_{j} v_{l}\right)+O(|u|+|z|)^{4} .
$$

On choisit $(V)=\left(V_{1}, \ldots, V_{r}\right)$ un système de coordonnées holomorphes sur $E$ au voisinage de $z_{0}$ tel que

$$
\|V\|_{H}^{2}=\sum_{\lambda}\left|V_{\lambda}\right|^{2}-\sum_{j k \lambda \mu} d_{j k \lambda \mu} u_{j} \bar{u}_{k} V_{\lambda} \bar{V}_{\mu}+O\left(|u|^{3}|V|^{2}\right),
$$

où $\left(d_{j k \lambda \mu}\right)$ est le tenseur de courbure de $(E, H)$. Un tel système est dit $H$-synchrone en $z_{0}$ des coordonnées géodésiques $(u)$. On appelle $\left(a_{\underline{\lambda}}^{0}\right)$ la matrice de la métrique $h_{0}$ dans ces coordonnées. 
Au voisinage de $z_{0}$, la métrique $h_{\varepsilon} \mathrm{s}^{\prime}$ écrit donc

$$
h_{\varepsilon}(z)(V)=\frac{1}{C \varepsilon^{2 n}} \int_{u \in \mathbf{C}^{n}} \chi^{\prime}\left(\frac{|\eta(z, u)|^{2}}{\varepsilon^{2}}\right) \sum_{\lambda \underline{\lambda}} a_{\lambda \underline{\lambda}}^{0}(u) \tau_{z, u}(V)_{\lambda} \overline{\tau_{z, u}(V)_{\underline{\lambda}}} d \lambda(\eta) .
$$

Sous cette forme, $h_{\varepsilon}$ apparaît comme une métrique de classe $C^{\infty}$.

Reste à montrer que pour $\varepsilon>0$ assez petit, $i c_{h_{\varepsilon}}(E)<_{\text {Griff }}-\nu \omega \otimes \operatorname{Id}_{E}$; c'est-à-dire par le lemme 4 que pour $\varepsilon>0$ assez petit, $i \partial \bar{\partial} h_{\varepsilon}(\cdot)(\cdot)>\nu h_{\varepsilon} p^{\star} \omega$, où $p: E \rightarrow X$ est la projection naturelle.

Pour le calcul du hessien de $h_{\varepsilon}(\cdot)(\cdot)$, on dérivera sous le signe intégral après avoir étudié la dépendance en $z$ et $V$ du transport parallèle $\tau$ et des coordonnées $(\eta)$. On transformera ensuite les dérivées en $(z)$ en des dérivées en $(u)$. Il apparaîtra le hessien de $h_{0}$, minoré par hypothèse. La continuité de $h_{0}$ suffira pour montrer que les autres termes tendent vers 0 avec $\varepsilon$.

On commence par établir un développement du transport parallèle le long de la géodésique $\gamma: t \mapsto \exp _{z} t \xi \simeq\left(u_{k}\right)=\left(z_{k}+t v_{k}+O\left(|z|+|u|^{3}\right)\right.$.

Puisque les coordonnées sur $E$ sont $H$-synchrones en $z_{0}$ des coordonnées (u), la matrice de la connexion $D^{H}$ est de la forme

$$
\xi\rfloor A^{D^{\mu}}(u)=-\sum_{j k \lambda \mu} d_{j k \lambda \mu} \xi_{j} \bar{u}_{k} e_{\mu}^{\star} \otimes e_{\lambda}+O(|u|)^{2}|\xi| .
$$

Le transport parallèle est donné par

$$
\left.\frac{d \gamma}{d t}\right\rfloor D^{H}(\tau V)=0
$$

soit

$$
\frac{d}{d t}(\tau V)_{\lambda}-\sum_{j k \mu} d_{j k \lambda \mu} v_{j}\left(\overline{z_{k}}+t \bar{v}_{k}\right)(\tau V)_{\mu}=O(|z|+|u|)^{3}
$$

Par intégration, on démontre que

$$
(\tau V)_{\lambda}=V_{\lambda}+\sum_{j k \mu} d_{j k \lambda \mu} v_{j}\left(\overline{z_{k}}+\frac{\overline{v_{k}}}{2}\right) V_{\mu}+O(|z|+|u|)^{3} .
$$

D' où

$$
\begin{aligned}
& (\tau V)_{\lambda} \overline{(\tau V)_{\underline{\lambda}}}=V_{\lambda} \bar{V}_{\underline{\lambda}}+\sum_{j k \mu} d_{j k \lambda \mu} v_{j}\left(\bar{z}_{k}+\frac{\bar{v}_{k}}{2}\right) V_{\mu} \bar{V}_{\underline{\lambda}} \\
& +\sum_{j k \underline{\mu}} \overline{d_{j k \underline{\mu} \underline{\mu}} \bar{v}_{j}}\left(z_{k}+\frac{v_{k}}{2}\right) V_{\lambda} \bar{V}_{\underline{\mu}}+O(|z|+|u|)^{3}|V|^{2} .
\end{aligned}
$$

Pour transformer les dérivations en $(z)$ en des dérivations en $(u)$ dans le calcul du hessien de $h_{\varepsilon}$, on introduit les opérateurs

$$
\nabla_{l}^{+}:=\frac{\partial}{\partial z_{l}}+\frac{\partial}{\partial u_{l}} \text { en particulier } \nabla_{l}^{+} v_{p}=0 \nabla_{l}^{+}{\overline{v_{p}}}=0
$$




$$
\nabla \bar{m}_{m}:=\frac{\partial}{\partial \bar{z}_{m}}-\frac{\partial}{\partial \bar{u}_{m}} \quad \nabla \bar{m} v_{p}=0 \nabla \bar{m} \bar{v}_{p}=-2 \delta_{p, m}
$$

La contribution du transport parallèle est

$$
\nabla_{l}^{+}\left((\tau V)_{\lambda} \overline{(\tau V)_{\underline{\lambda}}}\right)=\sum_{j \underline{\mu}} \overline{d_{j l \underline{\lambda}} \underline{\underline{\mu}}} \bar{v}_{j} V_{\lambda} \bar{V}_{\underline{\mu}}+O(|z|+|u|)^{2}|V|^{2} .
$$

A partir de l'expression précédente de $\eta_{k}$, on calcule

$$
\begin{aligned}
\left|\eta_{k}\right|^{2}=\mid & \left.v_{k}\right|^{2}-\frac{1}{2} \sum_{j l p} c_{j k l p}\left(\overline{z_{p}} u_{l}+\frac{1}{3} \bar{v}_{p} v_{l}\right) v_{j} \overline{v_{k}} \\
& \quad-\frac{1}{2} \sum_{j l p} \overline{c_{k j p l}}\left(z_{l} \overline{u_{p}}+\frac{1}{3} v_{l} \overline{v_{p}}\right) \bar{v}_{k} v_{j}+O(|u|+|z|)^{5} .
\end{aligned}
$$

Puisque $\overline{c_{k j p l}}=c_{j k l p}$, on obtient

$$
\nabla_{l}^{+}\left(\chi^{\prime}\left(\frac{|\eta|^{2}}{\varepsilon^{2}}\right)\right)=\frac{-\chi^{\prime \prime}}{2 \varepsilon^{2}} \sum_{j k p} c_{j k l p}\left(\overline{z_{p}}+\overline{u_{p}}\right) v_{j} \overline{v_{k}}-\frac{\chi^{\prime \prime}}{2 \varepsilon^{2}} O(|u|+|z|)^{4}
$$

D'après [Dem1]

$$
d \lambda(\eta)=\left(\frac{i}{2}\right)^{n} d \eta_{1} \wedge d \bar{\eta}_{1} \wedge \ldots \wedge d \eta_{n} \wedge d \bar{\eta}_{n}
$$

où $\eta$ est différentié par rapport à $u$ à $z$ fixé. En utilisant $c_{l k j p}=c_{j k l p}$ et $v_{k}=u_{k}-z_{k}$, on obtient

$$
d \lambda(\eta)=d \lambda(u)\left[1-\sum_{k l p} c_{k k l p}\left(u_{i} \overline{u_{p}}-\frac{1}{3} v_{l} \overline{v_{p}}\right)+O(|u|+|z|)^{3}\right] .
$$

Donc

$$
\nabla_{l}^{+}(d \lambda(\eta))=-d \lambda(u)\left[\sum_{k p} c_{k k l p} \bar{u}_{p}+O(|u|+|z|)^{2}\right] .
$$

Les trois calculs précédents donnent

$$
\begin{aligned}
& \nabla_{l}^{+}\left(\chi^{\prime} d \lambda(\eta)(\tau V)_{\lambda} \overline{(\tau V)_{\underline{\lambda}}}\right) \\
& \quad=\left[-\frac{\chi^{\prime \prime}}{2 \varepsilon^{2}} \sum_{j k p} c_{j k l p}\left(\overline{z_{p}}+\bar{u}_{p}\right) v_{j} \bar{v}_{k}+\frac{\chi^{\prime \prime}}{\varepsilon^{2}} O(|u|+|z|)^{4}\right] d \lambda(\eta)(\tau V)_{\lambda} \overline{(\tau V)_{\underline{\lambda}}} \\
& \quad-\chi^{\prime} d \lambda(u)\left[\sum_{k p} c_{k k l p} \overline{u_{p}}+O(|u|+|z|)^{2}\right](\tau V)_{\lambda} \overline{(\tau V)_{\underline{\lambda}}} \\
& \quad+\chi^{\prime} d \lambda(\eta)\left[\sum_{j \underline{\mu}} \overline{d_{j l \underline{\underline{\mu}} \underline{\mu}}} \bar{v}_{j} V_{\lambda} \bar{V}_{\underline{\mu}}+O(|z|+|u|)^{2}|V|^{2}\right] .
\end{aligned}
$$

En utilisant 


$$
\begin{aligned}
\nabla_{m} \bar{v}_{p} & =-2 \delta_{m p} \\
\nabla_{m}\left((\tau V)_{\lambda} \overline{(\tau V)_{\underline{\lambda}}}\right) & =O(|u|+|z|)|V|^{2} \\
\nabla_{m}(d \lambda(\eta)) & =O(|u|+|z|) \mid d \lambda(u) \\
\nabla_{m}|\eta|^{2} & =-2 v_{m}+O(|u|+|z|)^{3},
\end{aligned}
$$

on obtient

$$
\begin{aligned}
& \nabla_{m} \nabla_{l}^{+}\left(\chi^{\prime} d \lambda(\eta)(\tau V)_{\lambda} \overline{(\tau V)_{\underline{\lambda}}}\right) \\
& =\left[\frac{\chi^{\prime \prime \prime}}{\varepsilon^{4}} \sum_{j k p} c_{j k l p}\left(\overline{z_{p}}+\overline{u_{p}}\right) v_{j} \overline{v_{k}} v_{m}+\frac{\chi^{\prime \prime \prime}}{\varepsilon^{4}} O(|u|+|z|)^{5}\right. \\
& \left.+\frac{\chi^{\prime \prime}}{\varepsilon^{2}} \sum_{j p} c_{j m l p}\left(\overline{z_{p}}+\overline{u_{p}}\right) v_{j}+\frac{\chi^{\prime \prime}}{\varepsilon^{2}} O(|u|+|z|)^{3}\right] d \lambda(\eta)(\tau V)_{\lambda} \overline{(\tau V)_{\underline{\lambda}}} \\
& +\left[\frac{2 \chi^{\prime \prime}}{\varepsilon^{2}} \sum_{k p} c_{k k l p} \overline{u_{p}} v_{m}+\frac{\chi^{\prime \prime}}{\varepsilon^{2}} O(|u|+|z|)^{3}\right. \\
& \left.+\chi^{\prime} \sum_{k} c_{k k l m}+\chi^{\prime} O(|u|+|z|)\right] d \lambda(u)(\tau V)_{\lambda} \overline{(\tau V)_{\underline{\lambda}}} \\
& \quad-2\left[\frac{\chi^{\prime \prime}}{\varepsilon^{2}} \sum_{j \underline{\mu}} \overline{d_{j l \underline{\lambda}} \underline{\mu}} \bar{v}_{j} v_{m} V_{\lambda} \bar{V}_{\underline{\mu}}+\frac{\chi^{\prime \prime}}{\varepsilon^{2}} O(|u|+|z|)^{3}|V|^{2}\right. \\
& \left.+\chi^{\prime} \sum_{\underline{\mu}} \overline{d_{m l \underline{\lambda} \underline{\mu}} V_{\lambda}} \bar{V}_{\underline{\mu}}+\chi^{\prime} O(|u|+|z|)|V|^{2}\right] d \lambda(\eta) .
\end{aligned}
$$

Au point $z_{0}$, cette expression devient

$$
\begin{aligned}
& \nabla_{m} \nabla_{l}^{+}\left(\chi^{\prime} d \lambda(\eta)(\tau V)_{\lambda} \overline{(\tau V)_{\underline{\lambda}}}\right) \\
& \quad=\left[\frac{\chi^{\prime \prime \prime}}{\varepsilon^{4}} \sum_{j k p} c_{j k l p} \overline{u_{p}} u_{j} \overline{u_{k}} u_{m}+\frac{\chi^{\prime \prime}}{\varepsilon^{2}} \sum_{j p} c_{j m l p} \bar{u}_{p} u_{j}\right. \\
& \left.+\frac{2 \chi^{\prime \prime}}{\varepsilon^{2}} \sum_{k p} c_{k k l p} \bar{u}_{p} u_{m}+\chi^{\prime} \sum_{k} c_{k k l m}+O(\varepsilon)\right] d \lambda(u) V_{\lambda} \bar{V}_{\underline{\lambda}} \\
& \quad+\left[-2 \frac{\chi^{\prime \prime}}{\varepsilon^{2}} \sum_{j \underline{\mu}} \overline{d_{j l \underline{\underline{\lambda}} \underline{\mu}} \bar{u}_{j} u_{m}} \bar{V}_{\underline{\mu}}-2 \chi^{\prime} \sum_{\underline{\mu}} \overline{d_{m l \underline{\lambda}} \underline{\underline{\mu}}} V_{\underline{\mu}}+O(\varepsilon)|V|\right] d \lambda(u) V_{\lambda} .
\end{aligned}
$$

Afin d'intégrer par parties l'expression du hessien de $h_{\varepsilon}$ et d'utiliser la majoration de la courbure de $E$ muni de $h_{0}$, il faut reconnaître des dérivées secondes par rapport aux coordonnées $(u)$. Le terme nouveau par rapport au cas de la régularisation des fonctions plurisousharmoniques est 


$$
\begin{aligned}
& -2 \frac{\chi^{\prime \prime}}{\varepsilon^{2}} \sum_{j} \overline{d_{j l \underline{\lambda}} \underline{u} \bar{u}_{j} u_{m}}-2 \chi^{\prime} \overline{d_{m l \underline{\underline{\lambda}} \underline{\mu}}} \\
& \quad=-2 \sum_{j} \frac{\partial^{2}}{\partial u_{j} \partial \bar{u}_{m}}\left(\varepsilon^{2} \chi \overline{d_{j l \underline{\underline{\lambda}} \underline{\mu}}}\right)+O(\varepsilon),
\end{aligned}
$$

comme on peut le vérifier en utilisant

$$
\frac{\partial}{\partial u_{l}}|\eta|^{2}=\bar{u}_{l}+O(\varepsilon) \text {. }
$$

Les autres termes se réécrivent

$$
\begin{gathered}
\frac{\chi^{\prime \prime \prime}}{\varepsilon^{4}} \sum_{j k p} c_{j k l p} \overline{u_{p}} u_{j} \bar{u}_{k} u_{m}+\frac{\chi^{\prime \prime}}{\varepsilon^{2}} \sum_{j p} c_{j m l p} \overline{u_{p}} u_{j} \\
+\quad \frac{2 \chi^{\prime \prime}}{\varepsilon^{2}} \sum_{k p} c_{k k l p} \overline{u_{p}} u_{m}+\chi^{\prime} \sum_{k} c_{k k l m} \\
=\quad \sum_{p} \frac{\partial^{2}}{\partial u_{p} \partial \bar{u}_{m}}\left(\chi^{\prime} \sum_{j k} c_{j k l p} u_{j} \overline{u_{k}}+\varepsilon^{2} \chi \sum_{k} c_{k k l p}\right) \\
-\sum_{j k} \frac{\partial^{2}}{\partial u_{k} \partial \overline{u_{j}}}\left(\varepsilon^{2} \chi c_{j k l m}\right)+O(\varepsilon) .
\end{gathered}
$$

Puisque $\chi^{\prime} \sum_{\lambda \underline{\lambda}} a_{\lambda \underline{\underline{\lambda}}}^{0}(u)\left(\tau_{z, u} V\right)_{\lambda} \overline{\left(\tau_{z, u} V\right)_{\underline{\lambda}}} d \lambda(\eta)$ est une $(n, n)$-forme réelle sur $X$,

$$
\begin{aligned}
& \sum_{l m \underline{\lambda} \underline{\lambda}} a_{\lambda \underline{\underline{\lambda}}}^{0}(u)\left(\frac{\partial^{2}}{\partial z_{l} \partial \bar{z}_{m}}-\frac{\partial^{2}}{\partial u_{l} \partial \bar{u}_{m}}\right)\left(\chi^{\prime}\left(\tau_{z, u} V\right)_{\lambda} \overline{\left(\tau_{z, u} V\right)_{\underline{\lambda}}} d \lambda(\eta)\right) s_{l} \bar{s}_{m} \\
& =\operatorname{Re} \sum_{l m \underline{\lambda} \underline{\underline{\lambda}}} a_{\lambda \underline{\lambda}}^{0}(u) \nabla_{m} \nabla_{l}^{+}\left(\chi^{\prime}\left(\tau_{z, u} V\right)_{\lambda} \overline{\left(\tau_{z, u} V\right)_{\underline{\lambda}}} d \lambda(\eta)\right) s_{l} \bar{s}_{m} \\
& =\operatorname{Re}\left[\sum_{l m \underline{\lambda} \underline{\lambda} p} a_{\underline{\lambda}}^{0}(u) \frac{\partial^{2}}{\partial u_{p} \partial \bar{u}_{m}}\left(\chi^{\prime} \sum_{j k} c_{j k l p} u_{j} \bar{u}_{k}+\varepsilon^{2} \chi \sum_{k} c_{k k l p}\right) s_{l} \bar{s}_{m} V_{\lambda} \bar{V}_{\underline{\lambda}}\right. \\
& -\sum_{l m \lambda \underline{\lambda} j k} a_{\lambda \underline{\lambda}}^{0}(u) \frac{\partial^{2}}{\partial u_{k} \partial \overline{u_{j}}}\left(\varepsilon^{2} \chi c_{j k l m}\right) s_{l} \bar{s}_{m} V_{\lambda} \bar{V}_{\underline{\lambda}} \\
& \left.-2 \sum_{l m \underline{\lambda} \underline{\lambda} \underline{\mu} \underline{\mu}} a_{\lambda \underline{\underline{\lambda}}}^{0}(u) \frac{\partial^{2}}{\partial u_{j} \partial \bar{u}_{m}}\left(\varepsilon^{2} \chi \overline{d_{j l \underline{\lambda}} \underline{\mu}}\right) V_{\lambda} \bar{V}_{\underline{\mu}} s_{l} \bar{s}_{m}+O(\varepsilon)|s|^{2}|V|^{2}\right] d \lambda(u) \text {. }
\end{aligned}
$$

On peut alors intégrer par parties l'expression du hessien de $h_{\varepsilon}$ obtenue par dérivation sous le signe intégral.

$$
\begin{aligned}
\sum_{l m} & \frac{\partial^{2} h_{\varepsilon}(.)(V)}{\partial z_{l} \partial \overline{z_{m}}} s_{l} \bar{s}_{m} \\
\quad & \frac{1}{C \varepsilon^{2 n}} \int_{(u)} \sum_{l m} \chi^{\prime} \frac{\partial^{2} h_{0}(.)(V)}{\partial u_{l} \partial \bar{u}_{m}} s_{l} \bar{s}_{m} d \lambda(u) \\
& +\frac{1}{C \varepsilon^{2 n}} \operatorname{Re} \int_{(u)} \sum_{l m p} \frac{\partial^{2} h_{0}(.)(V)}{\partial u_{p} \partial \bar{u}_{m}}\left(\chi^{\prime} \sum_{j k} c_{j k l p} u_{j} \overline{u_{k}}+\varepsilon^{2} \chi \sum_{k} c_{k k l p}\right) s_{l} \bar{s}_{m} d \lambda(u)
\end{aligned}
$$




$$
\begin{aligned}
& -\frac{2}{C \varepsilon^{2 n}} \operatorname{Re} \int_{(u)}\left[\sum_{j l m \lambda \underline{\lambda} \underline{\mu}} \frac{\partial^{2} a_{\lambda \underline{\lambda}}^{0}}{\partial u_{j} \partial \overline{u_{m}}} \varepsilon^{2} \chi \overline{d_{j l \underline{\lambda}} \underline{\mu}} V_{\lambda} \bar{V}_{\underline{\mu}} s_{l} \bar{s}_{m}+O(\varepsilon)|s|^{2}|V|^{2}\right] d \lambda(u) \\
& -\frac{1}{C \varepsilon^{2 n}} \int_{(u)_{j k l m}} \frac{\partial^{2} h_{0}(.)(V)}{\partial u_{k} \partial \bar{u}_{j}} \varepsilon^{2} \chi c_{j k l m} s_{l} \bar{s}_{m} d \lambda(u) .
\end{aligned}
$$

La minoration du hessien de $h_{0}$ montre que le premier terme est supérieur à la quantité $(\nu+\alpha+O(\varepsilon))\|s\|_{\omega}^{2}\|V\|_{h_{\text {e }}}^{2}$

On montre maintenant que les autres termes tendent vers 0 avec $\varepsilon$. Puisque le calcul est local, on peut supposer, quitte à multiplier par un poids de classe $\mathscr{C}^{\infty}$, que

$$
\forall\left(u_{\lambda}\right),\left(s_{j}\right), \sum_{j m \lambda \underline{\underline{\lambda}}} \int_{(u)} \frac{\partial^{2} a_{\lambda \lambda_{\lambda}^{\lambda}}^{0}}{\partial u_{j} \partial \overline{u_{m}}} V_{\lambda} \bar{V}_{\underline{\lambda}} s_{j} \bar{s}_{m} d \lambda(u) \geq 0 .
$$

En utilisant une transformée de Fourier discrète, comme dans [D-S 80], on en déduit qu'il existe une constante $C$ telle que

$$
\begin{aligned}
\forall \lambda, \underline{\lambda}, j, m, \int_{(u)}\left|\frac{\partial^{2} a_{\lambda \underline{\lambda}}^{0}}{\partial u_{j} \partial \bar{u}_{m}}\right| d \lambda(u) & \leq C \int_{(u)} \sum_{k} \frac{\partial^{2} \sum_{\mu} a_{\mu \mu}^{0}}{\partial u_{k} \partial \bar{u}_{k}} d \lambda(u) \\
& \leq C \int_{(u)} \Delta\left(\sum_{\mu} a_{\mu \mu}^{0}\right) d \lambda(u) .
\end{aligned}
$$

Reste à appliquer le lemme suivant pour obtenir l'estimation de courbure.

Lemme 10. Soit u une fonction continue plurisousharmonique au voisinage de 0 dans $\mathbf{C}^{n}$. Soit $\nu_{z_{0}}: t \mapsto \frac{1}{t^{2 n-2}} \int_{\left|z-z_{0}\right|<t} \Delta u d \lambda(z)$.

Alors $\nu_{z_{0}}(t)$ est une fonction croissante qui tend vers 0 quand $t$ tend vers 0 , uniformément en $z_{0}$.

Ce lemme exprime le fait que le nombre de Lelong d'une fonction plurisousharmonique en un point de continuité est nul [Lel]. De plus, pour tout $t$, la fonction $z_{0} \mapsto \nu_{z_{0}}(t)$ est continue.

\subsection{Démonstration du théorème de positivité au sens de Griffiths}

\subsubsection{Première étape:}

On suppose que $Y$ satisfait l'hypothèse $(\mathrm{H})$. On suppose pour commencer que $L$ est nef. L'auto-application va permettre de rendre arbitrairement petite la perte de positivité. A partir de l'itérée $p$-ième de $\theta$, notée $\theta^{(p)}$, on construit le diagramme 


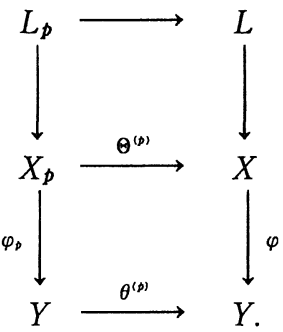

On note toujours $E:=\varphi_{\star}\left(K_{X / Y} \otimes L\right)$. A partir de la fonction $F$ satisfaisant

$$
\theta^{\star} \omega \geq a \omega+i \partial \bar{\partial} F(a:=\alpha-\varepsilon>1)
$$

on construit par récurrence sur $p$ une fonction $F_{p}$ satisfaisant

$$
\theta^{(p) \star} \omega \geq a^{p} \omega+i \partial \bar{\partial} F_{p}
$$

Puisque $\varphi$ est une submersion $\left(\Theta^{(p) \star} K_{X / Y}=K_{X_{p} / Y_{p}}\right)$ et que $\theta^{(p)}$ est fini donc plat, la formule de changement de base pour les fibrés adjoints donne $\theta^{(p) \star} E=$ $\left(\varphi_{p}\right)_{\star}\left(K_{X_{p} / Y} \otimes L_{p}\right)$. Le fibré $L_{p}=\Theta^{(p) \star} L$ est nef et $\varphi_{p}$-ample. On peut donc appliquer la proposition 1 aux données $\left(X_{p}, \varphi_{p}, L_{p}\right)$ : il existe une métrique hermitienne de classe $\mathscr{C}^{\infty} \operatorname{sur} \theta^{(p) \star} E$ telle que

$$
i c\left(\theta^{(p)} E\right) \geq_{\mathrm{Griff}}-A \omega \otimes \mathrm{Id} \geq_{\mathrm{Griff}}-A a^{-p}\left(\theta^{(p) \star} \omega-i \partial \bar{\partial} F_{p}\right) \otimes \mathrm{Id} .
$$

En multipliant cette métrique par $e^{A a^{-\triangleright} F_{p}}$, on obtient une métrique hermitienne de classe $\mathscr{C}^{\infty}$ sur $\theta^{(p) \star} E$ telle que

$$
{ }_{i c}\left(\theta^{(p) \star} E\right) \geq_{\text {Griff }}-A a^{-p} \theta^{(p) \star} \omega \otimes \operatorname{Id}
$$

et par la proposition 2, puisque $\theta^{(p)}$ est fini,

$$
i c(E) \geq_{\text {Griff }}-A a^{-p} \omega \otimes \text { Id. }
$$

On retrouve en particulier que $E=\varphi_{\star}\left(K_{X / Y} \otimes L\right)$ est numériquement effectif.

\subsubsection{Seconde étape:}

On suppose maintenant que $L$ est ample. On montre que $E:=\varphi_{\star}\left(K_{X / Y} \otimes L\right)$ est alors un fibré vectoriel strictement positif au sens de Griffiths sur $Y$.

Lemme 11. Soit $G$ un fibré en droites ample sur $Y$. Il existe un entier naturel p tel que $\Theta^{(p) \star} L \otimes \varphi_{p}^{\star} G^{-1}$ soit nef sur $X_{p}$.

Démonstration. On munit $L$ et $G$ de métriques hermitiennes de classe $\mathscr{C}^{\infty}$ à courbure strictement positive. On peut supposer, quitte à multiplier $\omega$, que $\omega \geq i c(G)$. Soit $\varepsilon>0$ tel que ic $(L) \geq \varepsilon \varphi^{\star} \omega+\varepsilon i c(L)$. Soit $p$ tel que $\varepsilon a^{p}>1$. 


$$
\begin{aligned}
& i c\left(\Theta^{(p)} L\right) \quad \geq \quad \varepsilon \varphi_{p}^{\text {ऐ }} \theta^{(p)} \omega+\varepsilon i c\left(\Theta^{(p) \text { स }} L\right) \\
& \geq \quad \varepsilon \varphi_{p}^{\bar{p}} a^{p} \omega+\varepsilon i \partial \bar{\partial} F_{p}{ }^{\circ} \varphi_{p}+\varepsilon i c\left(\Theta^{(p)} L\right)
\end{aligned}
$$

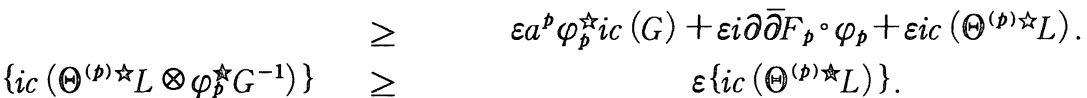

Puisque $\Theta^{(p)}$ est un morphisme fini, $\Theta^{(p)} L$ est ample et, par suite $\Theta^{(p)} L \otimes$ $\varphi_{p}^{\star} G^{-1}$ peut être muni d'une métrique hermitienne de classe $\mathscr{C}^{\infty}$ à courbure positive : il est donc nef.

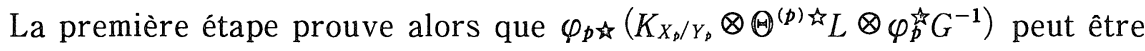
muni, pour tout $\varepsilon>0$, d'une métrique hermitienne de classe $\mathscr{C}^{\infty}$ dont la courbure vérifie

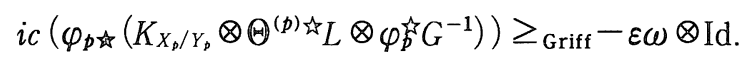

Par la formule de projection, on obtient que $\varphi_{p}\left(K_{X_{p} / Y_{p}} \otimes \Theta^{(p)} L\right)$ est strictement positif au sens de Griffiths. La formule de changement de base montre que ce dernier fibré vectoriel est isomorphe à $\theta^{(p)} E$. Puisque $\theta^{(p)}$ est un morphisme fini, on conclut grâce à la proposition 2 que $E=\varphi_{\downarrow}\left(K_{X / Y} \otimes L\right)$ est strictement positif au sens de Griffiths.

\section{§6. Applications}

\subsection{Variétés kählériennes compactes dont le fibré tangent est numériquement effectif}

Soit $X$ une variété kählérienne compacte dont le fibré tangent est numériquement effectif. D'après [DPS], il existe un revêtement étale fini $\widetilde{X} \rightarrow X$ tel que $\tilde{X}$ vérifie les mêmes hypothèses que $X$ et tel que $\widetilde{\alpha}$, le morphisme d'Albanese de $\widetilde{X}$, soit une submersion (sur un tore) dont les fibres sont des variétés de Fano. Le fibré anti-canonique (relatif) est nef et $\widetilde{\alpha}^{-}$ample. Par application du théorème 2 , on déduit que pour tout $k \in \mathbb{N}, \widetilde{\alpha}_{\star}\left(K_{\bar{X}}^{k}\right)$ est numériquement effectif.

\subsection{Positivité de $S^{k} E \otimes \operatorname{det} E$}

On démontre le corollaire 2. Soit $E \rightarrow Y$ un fibré vectoriel ample sur une variété projective lisse $Y$ satisfaisant l'hypothèse $(\mathrm{H})$. Soit $X:=\mathbb{P}(E)$ la variété des hyperplans de $E, p: X \rightarrow Y$ la projection naturelle. Par hypothèse, le fibré en droites $\mathscr{O}_{E}(1)$ canoniquement associé à $E$ est ample sur $X$. Il suffit de remarquer que $K_{X / Y}=\mathscr{O}_{E}(-r) \otimes p^{\star} \operatorname{det} E$, et donc que $p_{\star}\left(K_{X / Y} \otimes \mathscr{O}_{E}(r+k)\right)=S^{k} E \otimes \operatorname{det} E$. On applique alors le théorème 3 .

On suppose maintenant que $Y$ admet un morphisme surjectif fini $\rho: Y^{\prime} \rightarrow Y$ où $Y^{\prime}$ est une variété projective lisse satisfaisant l'hypothèse $(H)$. D'après la proposition 4.3 de [Hart1], $\rho^{\star} E$ est ample sur $Y^{\prime}$. Le premier cas montre que 
$S^{k} \rho^{\star}(E) \otimes \operatorname{det} \rho^{\star}(E)=\rho^{\star}\left(S^{k} E \otimes \operatorname{det} E\right)$ est strictement positif au sens de Griffiths. Reste à appliquer la proposition 2 pour conclure.

En utilisant d'autres variétés de drapeaux de $E$ comme dans [Dem2], ou des produits fibrés de copies de $\mathbb{P}(E)$ comme dans [Man2] ou encore des sommes de copies de $E$, on obtient sur les variétés vérifiant l'hypothèse $(\mathrm{H})$, la stricte positivité au sens de Griffiths de fibrés construits sur $E$ par d'autres représentations du groupe linéaire tensorisées par une puissance strictement positive du déterminant de $E$. Puisque la somme de fibrés strictement positifs au sens de Griffiths est strictement positive au sens de Griffiths, on en déduit en particulier la stricte positivité au sens de Griffths de $E^{\otimes k} \otimes(\operatorname{det} E)^{\text {rangE-1}}$.

\subsection{Remarque: Méthode algébrique}

Les variétés de Fano d'indice supérieur ou égal à la dimension sont, d'après un théorème de Kobayashi-Ochiai [Ko-Oc], l'espace projectif $\mathbb{P}^{n}$ de dimension $n$ et la quadrique non dégénérée $\mathbb{Q}^{n}$ de dimension $n$ plongée dans $\mathbb{P}^{n+1}$. Par simple application du critère de Castelnuovo-Mumford, on peut retrouver le corollaire précédent sur ces variétés.

On rappelle d'abord le théorème d'annulation de Griffiths [Griff], selon lequel

$$
\forall k \in \mathbb{N}, \forall i>0, H^{i}\left(Y, S^{k} E \otimes \operatorname{det} E \otimes L \otimes K_{Y}\right)=0
$$

pour tout fibré vectoriel ample $E \rightarrow Y$ et tout fibré en droites $L \rightarrow Y$ nef.

Dans le cas de l'espace projectif, il existe un fibré en droites $G:=\mathscr{O}(1)$ très ample sur $Y$ tel que $G^{n+1}=K_{Y}^{-1}$. Ceci donne bien

$$
\forall i>0, H^{\imath}\left(Y, S^{k} E \otimes \operatorname{det} E \otimes G^{-1} \otimes G^{-\imath}\right)=0 .
$$

Le fibré $S^{k} E \otimes \operatorname{det} E \otimes G^{-1}$ est donc globalement engendré. Par conséquent, $S^{k} E \otimes$ $\operatorname{det} E$ est strictement positif au sens de Griffiths, et son dual est même strictement négatif au sens de Nakano.

Dans le cas de la quadrique $Y=\mathbf{Q}^{n}, n \geq 2$, l'indice n'est que $n$; il faut donc justifier autrement l'annulation du $n^{\text {1eme }}$ groupe de cohomologie. On a dans ce cas $K_{Y}=G^{-n}$ avec $G=\left.\mathscr{O}_{\mathbf{P}^{n+1}}\right|_{\mathbf{Q}^{n}}$, et $S^{k} E$ est ample tandis que det $E \otimes G^{-1}$ est semi-positif: si $n \geq 3$, ceci résulte du fait que le groupe de Picard de la quadrique est engendré par $G$; pour $n=2$, le groupe de Picard de $\mathbf{Q}^{2} \simeq \mathbf{P}^{1} \times \mathbf{P}^{1}$ est $\mathbf{Z}^{2}$ avec $G=\mathscr{O}(1,1)$; tout fibré en droites ample est donc minoré par $G$. Par dualité de Serre, on trouve alors

$$
H^{n}\left(Y, S^{k} E \otimes \operatorname{det} E \otimes G^{-1} \otimes G^{-n}\right)=H^{0}\left(Y,\left(S^{k} E \otimes \operatorname{det} E \otimes G^{-1}\right)^{\star}\right) \star=0
$$

car $S^{k} E \otimes \operatorname{det} E \otimes G^{-1}$ est ample.

Puisque le fibré quotient universel sur les grassmanniennes peut jouer le rôle du fibré en droites très ample $G$ dans le critère de Castelnuovo-Mumford, 


\section{les résultats précédents restent vrais sur ces variétés [Man2].}

\section{Références}

[Dan] Danilov, V. I., The geometry of toric varieties, Russian Math. Surveys, 33 (1978), 97-154.

[Dem1] J. P. Demailly, Estimations $L^{2}$ pour l'opérateur $\bar{\partial}$ d'un fibré vectoriel holomorphe semi-positif au dessus d'une variété kählérienne complète, Ann. Sci. Ec. Norm. Sup., 15 (1982), 457-511.

[Dem2] - Vanishing theorems for tensor power of an ample vector bundle, Invent. Math., 91 (1988), 203-220.

[Dem3] - Regularization of closed positive currents and intersection theory, J. Algebraic Geom., 1 (1992), 361-409.

[D-S 80] Demailly, J. P. et Skoda, H., Relations entre les notions de positivité de P. A. Griffiths et de S. Nakano, Séminaire P. Lelong et H. Skoda, année 1978-79, Lecture Notes in Math., 822 (1980), 304-309.

[DPS] Demailly, J. P., Peternell Th. et Schneider, M., Compact complex manifolds with numerically effective tangent bundles, J. Algebraic Geom., 3 (1994), 295-345.

[E-V] Esnault, H. et Viehweg, E., Lectures on vanishing theorems, DMV Seminar, Band 20. Birkhäuser Verlag (1992).

[Fran] Frankel, R., Some remarks on positive vector bundles, J. Differential Geom., 14 (1979), 143-148.

[Fuj] Fujita, T., On Kähler fiber spaces over curves, J. Math. Soc. Japan, 30 (1978), 779-794.

[Griff] Griffiths, P. A., Hermitian differential geometry, Chern classes and positive vector bundles. In : Global analysis, Princeton: Princeton University Press, 1969.

[Hart1] Hartshorne, R., Ample vector bundles, Publ. Math. I.H.E.S, 29 (1966), 319-349.

[Hart2] - Algebraic Geometry, Springer Verlag, Berlin, 1977.

[Ig 54] Igusa, J., On the structure of a certain class of Kähler manifolds, Amer. J. Math., $\mathbf{7 6}(1954), 669-678$.

[Kaw1] Kawamata, Y., Characterization of abelian varieties, Compositio Math., 43 (1981), 253-276.

[Kaw2] - A generalization of Kodaira-Ramanujam's vanishing theorem. Math. Ann., 261 (1982), 43-46.

[Ko-Oc] Kobayashi, S. et Ochiai, T., Characterizations of complex projective spaces and hyperquadrics, J. Math. Kyoto Univ., 13(1973), 31-47.

[Kol] Kollár, J., Higher direct images of dualizing sheaves I, Ann. Math., 123 (1986), 11-42.

[Lel] Lelong, P., Fonctions plurisousharmoniques et formes différentielles positives. Dunod, Paris, Gordon \& Breach, New York, 1968.

[Man1] Manivel, L., Théorèmes d'annulation pour les fibrés associés à un fibré ample, Ann. Scu. Norm. Pisa, 4 (1992), 515-565.

[Man2] - Vanishing theorems for ample vector bundles, Prépublication de l'Inst. Fourier, 334 (1996), Invent. Math., 127 (1997) 401-416.

[Mumf] Mumford, D., Lectures on curves on an algebraic surface, Ann. of Math. Stud. 59, Princeton U. Press, Princeton, 1966.

[Vie1] Viehweg, E., Vanishing theorems, J. Reine Angew. Math., 335 (1982), 1-8.

[Vie2] - Weak positivity and the additivity of the Kodaira dimension for certain fiber spaces, Adv. Stud. Pure Math., 1 (1983), 329-353.

[Vie3] - Quasi-projective Moduli for polarized manifolds, Springer-Verlag, 1995. 